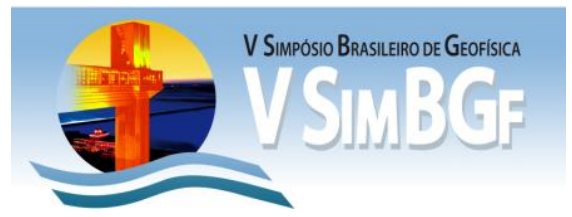

\title{
Mapas Tempo-Frequência de Alta Resolução Aplicados na Análise de Dados Sísmicos Time-Lapse
}

\author{
Wagner Moreira Lupinacci e Sérgio Adriano Moura Oliveira, LENEP/UENF
}

Copyright 2012, SBGf - Sociedade Brasileira de Geofísica

Este texto foi preparado para a apresentação no V Simpósio Brasileiro de Geofísica, Salvador, 27 a 29 de novembro de 2012. Seu conteúdo foi revisado pelo Comitê Técnico do V SimBGf, mas não necessariamente representa a opinião da SBGf ou de seus associados. É proibida a reprodução total ou parcial deste material para propósitos comerciais sem prévia autorização da SBGf.

\begin{abstract}
This work presents a comparative study, applied to time-lapse seismic, between the time-frequency maps obtained thru the use of continuous wavelet transform and the Choi-Willians energy density distribution. The latter method is theoretically capable of producing high resolution spectrograms. In order to verify the possibilities of these methods to detect and characterize subtle differences in the petro-seismic properties of the reservoir rock, induced by the fluid substitution process, we analyzed how this affects the local and instantaneous frequency attribute of the time-lapse seismic trace.
\end{abstract}

\section{Introdução}

$\mathrm{Na}$ literatura, podemos encontrar diferentes áreas de pesquisas que utilizam a decomposição espectral tempofrequência. Tais mapas permitem que seja feita uma analise da variação do conteúdo espectral do sinal em função do tempo. Na sísmica, esta técnica é largamente usada na exploração e monitoramento de hidrocarbonetos (Sinha et al., 2005; Wu e Liu, 2009; Liu e Fomel, 2012). Como exemplos mais específicos de aplicações destes mapas podemos citar a detecção direta de hidrocarbonetos (Castagna et al., 2003), visualização estratigráfica (Maklad e Dirstein, 2007) e sísmica time-lapse (Rojas, 2008). A representação da densidade de energia de um sinal em um mapa tempofrequência não é única e nenhum método é capaz de reproduzir o verdadeiro conteúdo de frequência do sinal em função do tempo. Dependendo da aplicação, cada método tem suas próprias vantagens e desvantagens.

Neste trabalho é realizado um estudo comparativo da decomposição espectral baseada na transformada contínua de wavelet e da decomposição espectral baseada na distribuição de Choi-Willians, que é um método teoricamente capaz de produzir mapas tempofrequência de resolução superior. Em seguida, o atributo frequência instantânea é calculado. Devido este ser um atributo muito instável, propõe-se a estimativa de uma frequência "local" derivada a partir dos mapas tempofrequência. Este atributo é então usado para analisar a resposta da mudança da impedância e do fator $q$ entre um dado base e o dado monitor.

\section{Modelagem sísmica time-lapse}

A onda sísmica sofre atenuação na medida em que se propaga. A substituição de fluidos que ocorre em um reservatório em produção altera a impedância sísmica e o fator de qualidade da rocha. A atenuação provoca queda de amplitude do pulso e perda de informações de alta frequência. Este efeito pode ser modelado como função da frequência, do duplo tempo de viajem da onda até o receptor e do fator de qualidade do meio (Wang, 2008). Para modelarmos estes efeitos no traço sísmico consideramos a incidência normal de uma onda plana compressional em um modelo de camadas horizontais (modelagem 1-D). O traço sísmico base é mostrado na figura 1. Para modelar o traço monitor consideraram-se as mudanças que ocorrem em uma pequena faixa (região delimitada pelas retas verdes). O efeito da variação da amplitude do traço base em $10 \%$ pode ser visualizado na figura 1.c. $O$ efeito devido à mudança no fator de qualidade do meio é exibido na figura 1.d. Observe que este efeito não se restringe somente à área produzida.

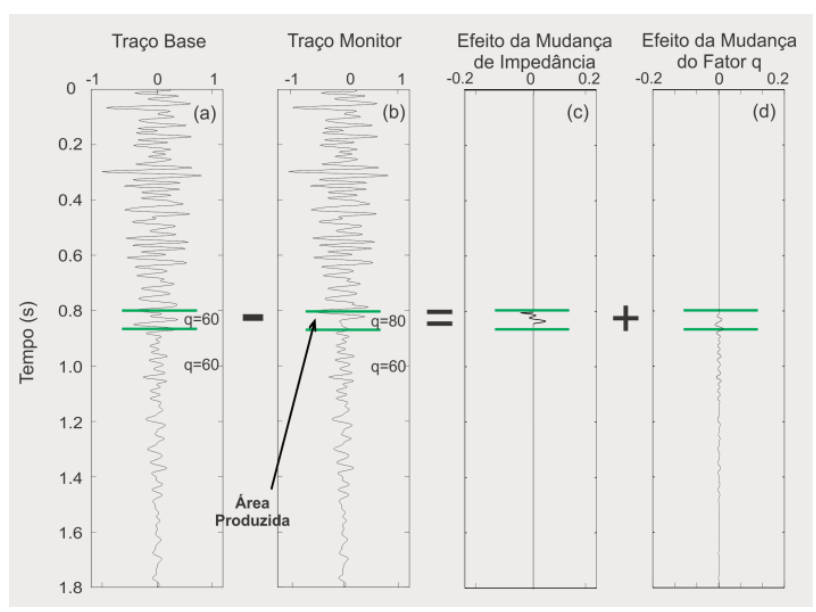

Figura 1 - (a) Traço sísmico base; (b) traço sísmico monitor; (c) e (d) diferença entre o traço monitor e o traço base devido a variação da amplitude do traço base e a mudança do fator $q$ de 60 para 80 dentro da região limitada pelas retas verdes, respectivamente.

\section{Mapas Tempo-Frequência}

A distribuição tempo-frequência de um sinal cria um mapa de densidade de energia em função das variáveis tempo e frequência. Para isto, existem diversos métodos. Neste trabalho foi analisada a transformada contínua de wavelet, baseada na função base de Morlet (TCM) e a 
distribuição de Choi-Willians (DCW), que produz um mapa de alta resolução espectral, pois não está sujeita à dicotomia entre a resolução temporal e a resolução espectral das transformadas lineares (ver, por exemplo: Mallat, 2009).

$\mathrm{Na}$ primeira análise do dado time-lapse, calculou-se o espectrograma usando TCM do traço base (figura 2.a), do traço monitor (figura 2.b) e o espectrograma da diferença entre o traço monitor e o traço base (figura 2.c). Podemos observar nesta figura que na região onde supostamente ocorreu substituição de fluido foi detectado uma anomalia.

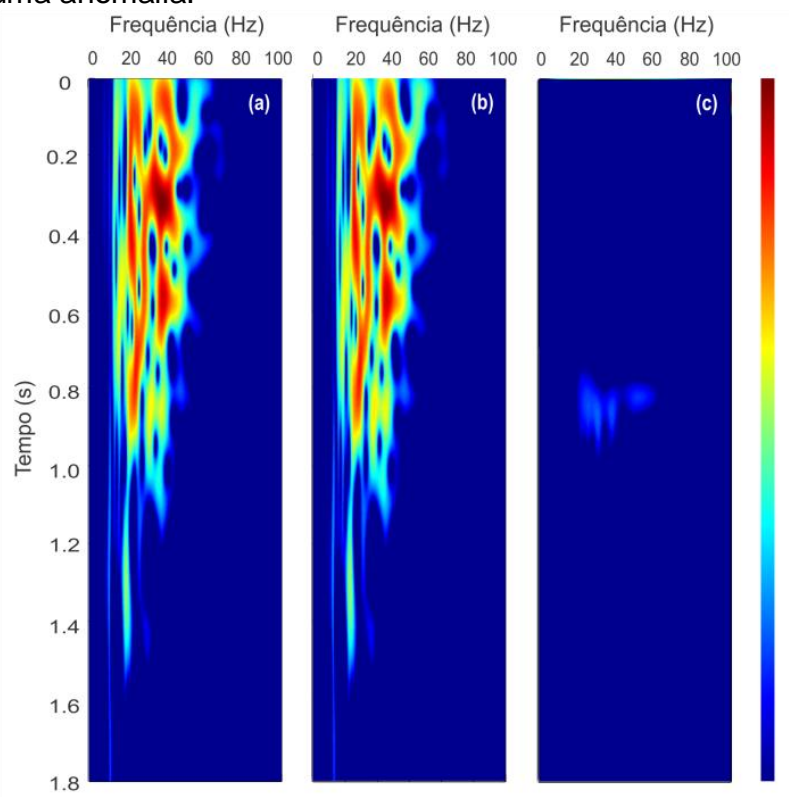

Figura 2 - (a), (b) e (c) representam os espectrogramas do traço base, do traço monitor da diferença entre o traço monitor e o traço base usando a transformada contínua de Morlet, respectivamente.

Os mesmos mapas tempo-frequência da figura 2 foram obtidos usando a DCW, figura 3. Ao utilizar a DCW, podemos perceber que as anomalias presentes nos mapas tempo-frequência são ainda mais focalizadas e concentradas na região limitada pelas retas verdes visualizadas na figura 1.

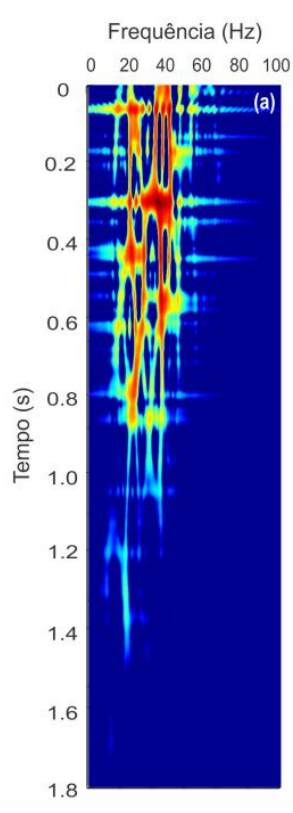

Frequência $(\mathrm{Hz})$
$0 \quad 20 \quad 40 \quad 60 \quad 80 \quad 100$

Frequência $(\mathrm{Hz})$

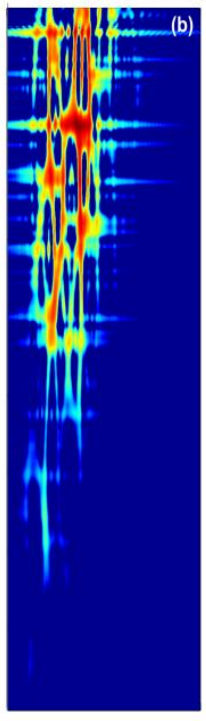

$\begin{array}{llllll}0 & 20 & 40 & 60 & 80 & 100\end{array}$

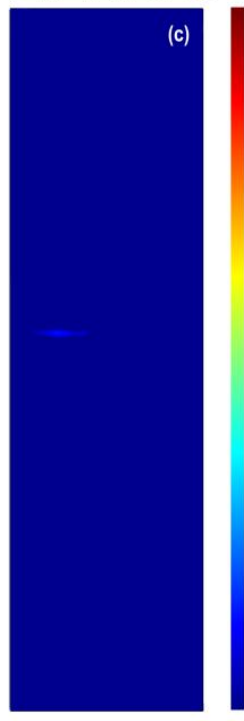

Figura 3 - (a), (b) e (c) representam os mapas tempofrequência do traço base, do traço monitor da diferença entre o traço monitor e o traço base usando a distribuição de Choi-Willians, respectivamente.

Outras características a serem analisadas são as anomalias provocadas pela diferença entre o traço monitor e o traço base com um acréscimo de $10 \%$ na amplitude do dado monitor para simbolizar uma mudança na impedância acústica e a variação do fator $q$ de 60 para 80 na região em análise, separadamente. Na figura 4 são apresentados os mapas tempo-frequência das diferenças entre 0 dado base e o dado monitor para cada caso usando a TCM e DCM. Na Figura 4.a, o espectrograma calculado usando a TCM apresentou uma anomalia que começou a ser perceptível antes que realmente a variação da impedância acontecesse. No caso do mapa tempo-frequência obtido usando a DCW, a anomalia é melhor focalizada, ou seja, este método apresentou uma melhor resolução temporal e espectral (figura 4.c) ao analisar a variação da impedância acústica.

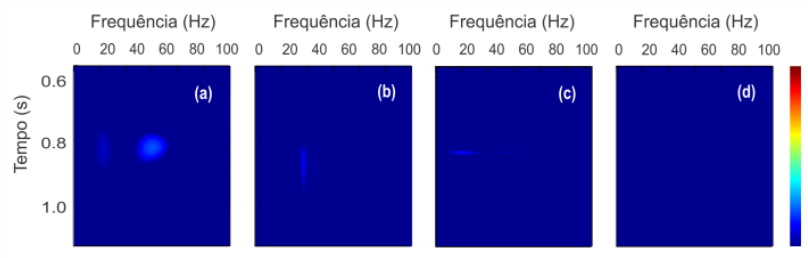

Figura 4 - (a) e (b) representam os mapas tempofrequência usando a TCM da diferença entre o traço monitor e o traço base devido a variação da impedância e a mudança do fator $q$, respectivamente; (c) e (d) representam os mapas tempo-frequência usando a DCW da diferença entre o traço monitor e o traço base devido a variação da impedância e a mudança do fator $q$, respectivamente.

Com a variação do fator $q$, a anomalia no mapa tempo-frequência usando TCM começou a acontecer exatamente onde o fator $q$ foi substituído, figura 4.b. Por outro lado, ao utilizar a DCW para obter o mapa tempo- 
frequência a mudança do fator $q$ não foi perceptível, figura 4.d.

Para analisar a influência da variação da impedância acústica e do fator $q$ separadamente, considere duas camadas: uma com baixa impedância e outra subjacente com uma maior impedância e preenchida de gás no espaço poroso. Se ocorrer uma substituição de gás por água na camada inferior, o que se espera é que ocorra um aumento tanto na impedância acústica quanto no fator $q$ e a tendência é que estes dois efeitos provoquem um aumento no valor absoluto da amplitude sísmica, ou seja, os dois efeitos se somaram.

\section{Frequência Instantânea e Local}

Os atributos sísmicos fornecem importantes características do sinal sísmico. Estes atributos podem ser classificados em instantâneos ou locais. Os atributos instantâneos geralmente são muito instáveis, o que dificulta suas análises. Os atributos locais conseguem medir características do sinal nas vizinhanças de cada ponto. Estes atributos têm diversas aplicações na caracterização e monitoramento de reservatórios como, por exemplo, na detecção de canais (Liu e Marfurt, 2007) e anomalias de baixa frequência (Liu et al., 2011).

Para calcular a frequência instantânea, considere um traço sísmico $s(t)$, o correspondente traço sísmico complexo $c(t)$ é definido como:

$$
c(t)=s(t)+i H(t)
$$

no qual $H(t)$ é a transformada de Hilbert do traço real $s(t)$. O traço complexo também pode ser representado em termos de amplitude $A(t)$, e fase instantânea $\varphi(t)$ :

$$
c(t)=A(t) e^{i \varphi(t)}
$$

Por definição, a frequência instantânea $f i(t)$ é a derivada no tempo da fase instantânea:

$$
f i(t)=\frac{1}{2} \frac{\partial \varphi(t)}{\partial t}
$$

A estimativa desta frequência é altamente sensível à presença de ruído. A figura 5 mostra um traço sísmico e a frequência instantânea deste traço. Podemos observar como este atributo sísmico é bastante instável, o que dificulta a sua análise.

A curva azul da Figura 5.b mostra o resultado da suavização da frequência instantânea e podemos notar um decaimento desta frequência com o tempo. Isto é o que geralmente acontece nos dados sísmicos, porque a terra funciona como um filtro passa baixa durante a propagação do pulso sísmico. Com a suavização, importantes informações são perdidas como, por exemplo, a mudança da curva de decaimento da frequência "local" que tem relação com o tipo de rocha presente.

No caso de estudos relacionados com conteúdos locais, recomenda-se a análise de um atributo sísmico mais estável. Além disso, quanto menor for a necessidade de utilizar algum tipo de filtragem, maior será o número de informações locais presentes no dado.

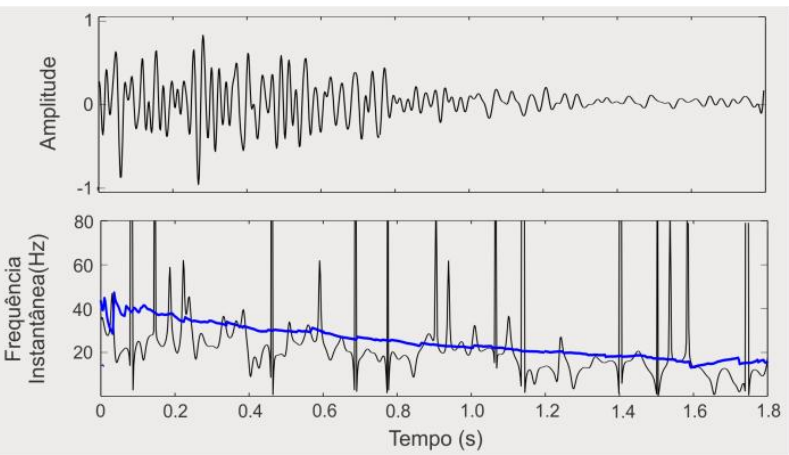

Figura 5 - (a) Traço sísmico sintético; (b) freqüência instantânea (curva preta) e freqüência instantânea suavizada (curva azul) do traço sísmico da letra a.

A frequência "local" é um atributo sísmico menos oscilatório do que a frequência instantânea e foi proposto pela primeira vez por Fomel, 2007. Neste trabalho, eu proponho uma forma alternativa de calcular este atributo. Para obtê-lo, primeiro é necessário calcular a distribuição de energia do sinal $D(t, f)$. Nesta etapa, diversos métodos podem ser analisados.

Com a distribuição da energia calculada, a frequência "local" $f l(t)$ é a primeira frequência que faz com que a soma da distribuição da energia seja superior a metade de toda a energia do sinal no instante de tempo $t$, ou seja, o primeiro elemento $f_{i}$ que satisfaz a condição:

$$
\sum_{i=1}^{n} D\left(t_{\text {const }}, f\right) \geq \frac{D_{T}}{2} \rightarrow f l=f_{i}
$$

será a frequência "local". $D_{T}$ representa a energia total no tempo $t$, fi a frequência de pico e $n$ o número de amostras do traço sísmico.

\section{Resultados}

As frequências "locais" estimadas usando a TCM e a CWD apresentaram características distintas. Com a TCM, obteve-se uma curva da frequência "local" mais suavizada, como podemos observar na figura 6.a. As figuras 6.b, 6.c e 6.d mostram os resultados da diferença entre as frequências "locais" do dado monitor pelo dado base com a mudança em conjunto da impedância e do fator $q$, com a mudança da impedância e com a mudança do fator $q$, respectivamente. Nestes casos, podemos observar que estes efeitos provocaram uma pequena alteração na frequência "local" e a variação máxima da frequência de pico foi de aproximadamente $3 \mathrm{~Hz}$.

Com o método DCW, a estimativa da frequência "local" apresentou ser mais instável (Figura 7.a). A diferença entre as frequências "locais" entre o traço monitor e o traço base com a variação em conjunto e separadamente da impedância e do fator $q$ produziram uma variação maior do que $8 \mathrm{~Hz}$ (Figuras 7.b, 7.c e 8.d).

A Figura 8 mostra a comparação da frequência local obtida usando a TCM (curva preta) e a DCW (curva azul). A alteração da impedância e do fator $q$ causaram poucas modificações, curva preta na figura 8.b. Por outro lado, os efeitos das mudanças destes parâmetros na área produzida resultou em maiores alterações na freqüência "local", curva azul na figura 8.b. 


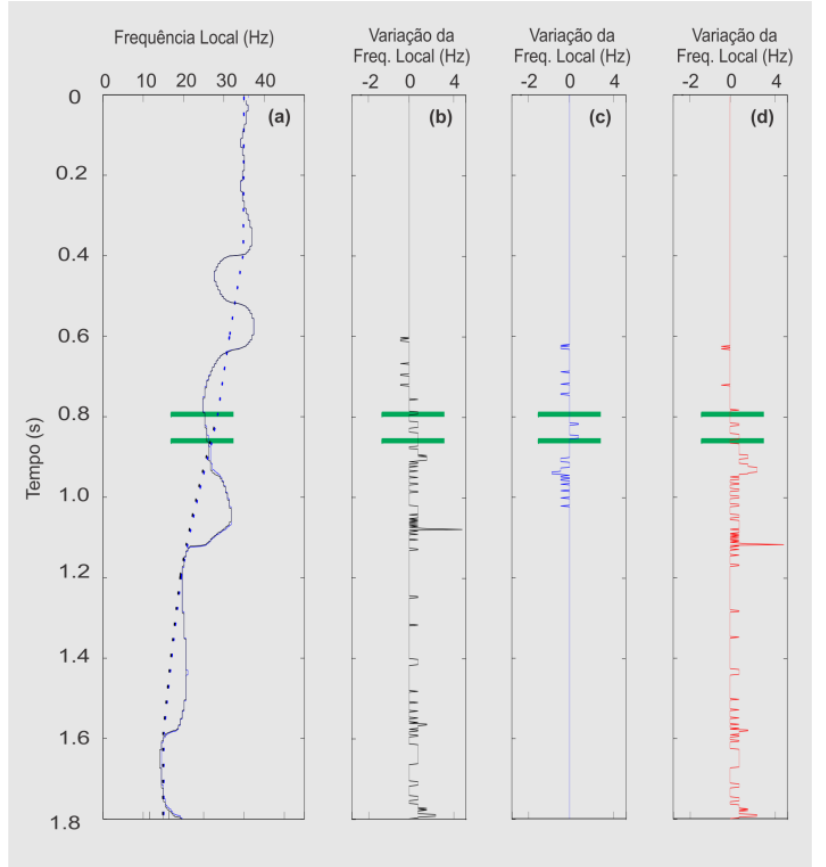

Figura 6 - (a) A frequência local do modelo é a curva tracejada e a frequência estimada usando a TCM é a curva contínua; (b), (c) e (d) diferença entre as frequências "locais" do traço monitor pelo traço base com o efeito da mudança em conjunto da impedância e do fator $\boldsymbol{q}$, somente com a mudança da impedância e somente com a mudança do fator $\boldsymbol{q}$, respectivamente.

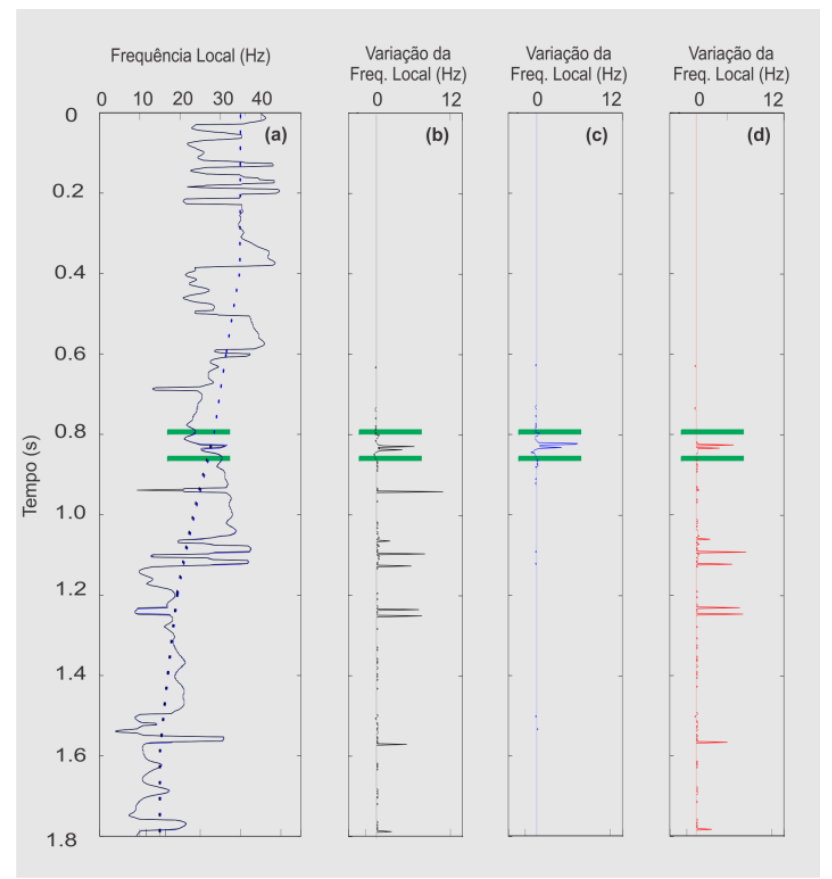

Figura 7 - (a) A frequência local do modelo é a curva tracejada e frequência estimada usando a DCW é a curva contínua; (b), (c) e (d) são as diferenças entre as frequências "locais" do traço monitor pelo traço base com o efeito da mudança em conjunto da impedância e do fator $q$, somente com a mudança da impedância $e$ somente com a mudança do fator $q$, respectivamente.

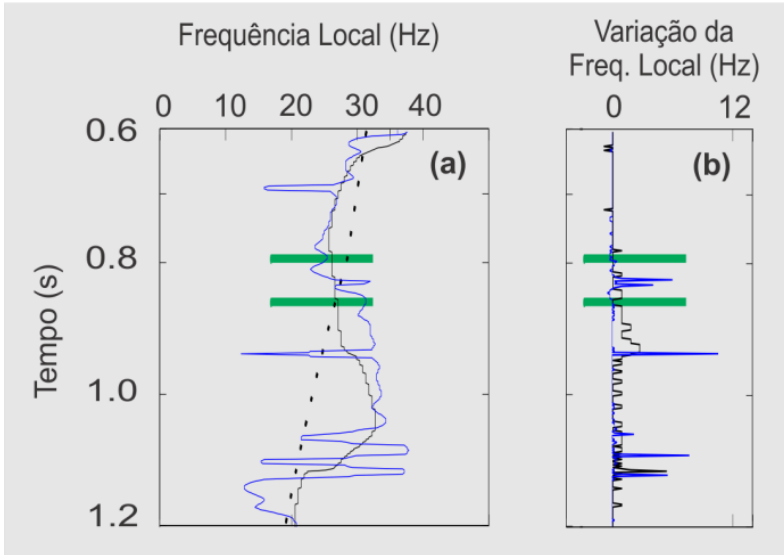

Figura 8 - (a) Representa as frequências "locais" estimadas do traço base usando a TCM (curva preta) e a DCW (curva azul); (b) diferença entre as frequências "locais" do traço monitor pelo traço base usando a TCM (curva preta) e a DCW (curva azul).

\section{Discussão e Conclusões}

Os mapas tempo-frequência calculados usando o método TCM apresentaram um decaimento mais constante do conteúdo de frequência do sinal quando comparado com a DCW. Sugerindo que a TCM são mais indicados em aplicações de balanceamento de energia do dado. A curva da frequência "local" estimada usando a TCM assemelha-se mais a curva de decaimento da frequência de pico e mostrou ser menos susceptíveis as variações no traço sísmico. Se conseguirmos medir este decaimento é possível modelar o efeito da atenuação usando o fator $q$.

Os eventos sísmicos apareceram mais focalizados nos mapas tempo-frequência quando a distribuição de energia do traço sísmico foi calculada com a DCW. Na análise dos dados, pode-se perceber o grande potencial desta transformada na aplicação para a determinação de limites de camadas delgadas. A frequência "local" estimada com a DCW apresentou ser mais instável. Neste caso, esta frequência é bem sensível as mudanças no traço sísmico o que torna este atributo uma interessante ferramenta na análise de dados time-lapse.

\section{Agradecimentos}

Os autores agradecem ao Laboratório de Engenharia e Exploração de Petróleo/Universidade Estadual Norte Fluminense (LENEP/UENF) e a CGGVeritas pelo apoio financeiro.

\section{Referências}

Castagna, J. P., Sun, S., Siegfried, R. W., 2003. Instantaneous spectral analysis: Detection of lowfrequency shadows associated with hydrocarbons. The Leading Edge, vol. 22: 120-132.

Fomel, S., 2007. Local seismic attributes. Geophysics, vol. 72: A29-A33.

Kjartansson, E., 1979. Constant Q-wave propagation and attenuation. Journal, vol. 84: 4737-4748. 
Liu, G., Fomel, S., Chen, X., 2011. Time-frequency analysis of seismic data using local attributes. Geophysics, vol. 76: P23-P34.

Liu, J., Marfurt, K. J., 2007. Instantaneous spectral attributes to detect channels. Geophysics, vol. 72: P23P31.

Liu, Y., Fomel, S., 2012. Seismic data using local timefrequency decomposition. Geophysical Prospecting, 110.

Maklad, M. S., Dirstein, J. K., 2007. Spectral detection of attenuation and lithology. Flow of Ideas, Hydrocarbons and Business. CSPG CSEG Convention, 501-505.

Mallat, S. G. 2009, A Wavelet Tour of Signal Processing, Third edition.

Academic Press, San Diego.

Rojas, N. A., 2008. Spectral decomposition applied to time-lapse seismic interpretation at Rulison field, Garfield County, Colorado. Dissertação - Colorado School of Mines.

Sinha, S., Routh, P. S., Anno, P. D., Castagna, J. P., 2005. Spectral decomposition of seismic data with continuous-wavelet transform. Geophysics, vol. 70: 1925.

Wang, Y., 2008. Seismic Inverse Q Filtering. Blackwell.

Wu, X., Liu, T., 2009. Spectral decomposition of seismic data with reassigned smoothed pseudo Wigner-Ville distribution. Journal of Applied Geophysics, vol. 68: 386393. 